\title{
Pricing, learning, and strategic behavior in a single-sale model ${ }^{\star}$
}

\author{
Hector Chade $^{1}$ and Virginia Vera de Serio $^{2}$ \\ 1 Department of Economics, Arizona State University, Main Campus, PO Box 873806, \\ Tempe, AZ 85287-3806, USA (e-mail: hector.chade@asu.edu) \\ 2 Facultad de Ciencias Económicas, Universidad Nacional de Cuyo, 5500 Mendoza, ARGENTINA \\ (e-mail: vvera@raiz.uncu.edu.ar)
}

Received: January 6, 1999; revised version: July 15, 2000

Summary. We analyze an infinite horizon model where a seller who owns an indivisible unit of a good for sale has incomplete information about the state of the world that determines not only the demand she faces but also her own valuation for the good. Over time, she randomly meets potential buyers who may have incentives to manipulate her learning process strategically. We show that i) the seller's incentives to post a high price and to experiment are not necessarily monotonic in the information conveyed by a buyer's rejection; and ii) as the discount factors tend to one, there are equilibria where the seller always ends up selling the good at an ex-post individually rational price.

Keywords and Phrases: Information revelation, Asymmetric information, Experimentation.

JEL Classification Numbers: C78, D82, D83.

\section{Introduction}

The problem studied in this paper can be illustrated as follows. Consider an individual who has a single indivisible item for sale but does not know whether its quality is high or low. If it is high, she knows that finding buyers who, in principle, are willing to pay a high price for it will be relatively easy; moreover, her own valuation for the item will be high, and she will refuse to trade it at a price below that value. That is, she would rather keep the good instead of underselling it. The opposite situation arises if its quality is low. In other words, the demand and the seller's valuation depend on the quality of the item, which is

\footnotetext{
* We thank Jorge Aseff, Alejandro Manelli, Mike Ormiston, Ed Schlee, and Joel Watson for their comments. The present version owes a considerable amount to the exceptionally helpful remarks and suggestions of an anonymous referee. The usual disclaimer applies.

Correspondence to: $\mathrm{H}$. Chade
} 
unknown to her. To learn more about it, she could meet buyers over time, offer the good at a high price, observe their actions, and then update her assessment about its quality. This is a costly strategy because she would rather sell it sooner than later, but it may allow the seller to improve her information and make a better decision. However, the problem can be complicated by the fact that buyers who have a high valuation for the item, and who are likely to meet her again in the future, may have incentives to 'play tough' and reject a high but still affordable price, so as to induce the seller to believe that the quality is low, and therefore make her more inclined to post a lower price in the future.

To capture the main features of this problem, we consider an infinite horizon single-sale model where the demand and the seller's valuation for the good depend on the state of the world that is unknown to her, and she meets potential buyers over time who behave strategically. In this environment with asymmetric information, active learning, and strategic behavior, we characterize the Perfect Bayesian Equilibria of the game and obtain two main results. First, we prove that, even if the buyers do not attempt to manipulate the seller's learning process, her incentives to post a high price and to experiment are not necessarily monotonic in the information conveyed by a buyer's rejection to buy at a high price; the intuition is that a change in the informativeness of this signal affects not only future beliefs, but also the 'weight' that the seller assigns to the payoff of selling in the future, and these two effects can work in opposite directions. Second, we show that, as the discount factors of the seller and the buyers tend to one, there are equilibria where the seller never trades at the 'wrong' price in the limit; i.e., in each state of the world, she ends up selling the good at an ex-post individually rational price. The main forces that drive this result are a) the existence of heterogeneous buyers in each state of the world, and b) the assumption that the number of buyers is greater than the number of sellers.

This paper is a contribution to a literature started by Wolinsky (1990), and further developed by Serrano and Yosha (1993, 1996), and Blouin and Serrano (1998). Wolinsky (1990) studies a model with active learning very similar to ours, but with a large number of buyers and sellers that meet pairwise in each period, and with informed and uninformed agents on each side of the market. He characterizes the steady-state equilibria of the model and shows that, as agents become almost infinitely patient then in any equilibrium a nonegligible fraction of uninformed agents trades at the 'wrong' price. Serrano and Yosha (1993) conduct the same analysis but under the assumption that one side of the market contains only informed agents; they find that Wolinsky's result is reversed in this case: in particular, they show that there is always a steadystate equilibrium where trade occurs at the 'right' price. Their subsequent paper contains a welfare analysis of these environments, demonstrating an equivalence between asymptotically ex-post individually rational and asymptotically (ex-ante, interim, and ex-post) efficient equilibrium sequences. Finally, Blouin and Serrano (1998) drop the steady-state assumption and focus on the one time entry case: they show that, in the limit, a nonnegligible fraction of uninformed agents trades at the 'wrong' price, and this holds whether informed agents are present on one 
or on both sides of the market. Our paper is closest to Blouin and Serrano (1998); in fact, we consider a simple model with one time entry, pairwise meetings, and informed agents on one side of the market. Unlike Blouin and Serrano's model, ours assume posted prices, heterogeneity of buyers' valuations in each state, and different number of agents on each side. As we explained above, the last two assumptions allow us to reverse the negative result obtained by Blouin and Serrano and show that there exist equilibria that exhibit trade at the right price in the limit.

This work is also related to the seminal paper by Rothschild (1974) that started the literature on experimentation in economics. ${ }^{1}$ The seller's problem analyzed in Section 3 amounts to solving a single-agent experimentation problem; the tractability of our framework allows us to characterize the seller's optimal policy, her incentives to experiment, and the asymptotic properties of the model in an elementary way. Moreover, the experimentation problem exhibits what Datta, Mirman, and Schlee (1998) called 'signal dependence'; i.e., the signal observed affects future payoffs not only through changes in beliefs. We provide an intuitive characterization of the different effects that this property has on the seller's behavior as the signal becomes more informative in Blackwell's sense, which allows us to explain why the degree of experimentation is nonmonotonic in this variable.

Other papers that have analyzed related issues regarding strategic pricing and learning in markets are Lazear (1986), Bergemann and Valimaki (1996), and Taylor (1999). Lazear (1986) also analyzes a single-sale model, where a seller faces a population of buyers whose common valuation for the good is unknown to her; he characterizes the seller's optimal pricing policy in the finite horizon case. Our model can be interpreted as an infinite horizon version of his, with two possible price announcements, noisy observations and a state-dependent seller's valuation; however, we also shed some light on an issue Lazear left unanswered: the effect that the strategic behavior of buyers has on the seller's optimal pricing policy. Bergemann and Valimaki (1996) study a model of a buyer who buys repeatedly from sellers who offer goods of different and unknown quality and compete in a Bertrand fashion; they focus on the stationary equilibria of the game and characterize the effect that price competition has on the buyer's optimal experimentation policy. Unlike their model, we consider a case where the seller extracts information about a single parameter and only trades once; moreover, we also analyze the incentives to experiment and the asymptotic properties of the model. Taylor (1999) considers the case where a seller optimally sets prices to manipulate how much buyers learn about quality; he pursues the analysis under different assumptions about the observable history of the game, but always allowing buyers to play only once and never face the seller again. Like his paper, ours also uses posted prices as the selling mechanism; however, we focus on the seller's learning process and allow buyers to meet her repeatedly.

\footnotetext{
${ }^{1}$ See also Mirman, Samuelson and Urbano (1993), Easley and Kiefer (1988), and the references cited therein.
} 
The paper is organized as follows. Section 2 describes the model. The main results are presented in Section 3. We first characterize the Perfect Bayesian Equilibrium of the game when buyers do not have incentives to manipulate the seller's learning process (we call it the 'impatient buyers' case), and we analyze the seller's incentives to announce a high price and experiment as a function of several parameters of the model. Then we study the case with 'patient buyers' who may have incentives to reject a high price, hoping that the seller will post a lower price in their next meeting. Finally, we analyze the asymptotic properties of the model as the discount factors of the seller and the buyers converge to one. Section 4 concludes. Most of the proofs are collected in the Appendix.

\section{The model}

Consider an individual who has an indivisible unit of a good for sale and who searches for potential buyers. The state of the world that prevails is unknown to her, and it can be either high or low; i.e., $\theta \in\{H, L\}$. She believes that it is $H$ with probability $0 \leq \mu_{0} \leq 1$. In the low state, she would be willing to sell at any price above $c_{L}$, while the minimum price she would accept in the high state is $c_{H}$. Whatever the state may be, there are two possible prices at which a transaction can take place: $p_{h}$ or $p_{l}$, with $p_{h}>c_{H}>p_{l}>c_{L}>0$.

There are $M$ buyers in each state of the world, and they can be of two different types: the $B$-buyers, whose valuation for the good is $B>p_{h}$ and who are, in principle, willing to buy at either price, and the $b$-buyers,

$p_{l}<b<p_{h}$, who only buy if the low price is posted. Unlike the seller, buyers know the state of the world. The proportions of buyers of each type are different in each state of the world: in the high state, the probability of meeting a $B$ is $\alpha$; in the low state, this probability is $\epsilon<\alpha$. Notice that this differs from the 'one-sided information case' with common values uncertainty considered in Serrano and Yosha (1993) and in Blouin and Serrano (1998), where agents on the informed side of the market were all of the same type in state $H$ and similarly in state $L$. In this sense, our set-up is slightly more general and combines elements of private and common values; moreover, it subsumes the case with common values (i.e., $\alpha=1$ and $\epsilon=0$ ) as a special case. This extra generality allows us to study the seller's incentives to post a high price and to experiment as a function of the informativeness of the observations (changes in $\alpha$ or $\epsilon$ ), and it also affects the limit properties of the model. ${ }^{2}$ In the sequel, we will carefully point out how the results change if $\alpha=1$ and $\epsilon=0$.

The timing of the events is the following: in each period, the seller randomly meets a buyer and, without observing his valuation, she announces a price. After the announcement the buyer decides whether to buy or not $(A=$ accept, $R=$ reject). If he accepts, the transaction takes place at the price posted by the seller

\footnotetext{
${ }^{2}$ See the discussion in Section 3.4.
} 
and the game ends; if he rejects, then the game continues and the seller is matched anew in the next period. ${ }^{3}$

The seller discounts payoffs using $0<\beta<1$; in other words, if she trades after $t$ periods at price $p_{i}, i=l, h$ and the true state is $\theta=H, L$, she enjoys $\beta^{t}\left(p_{i}-c_{\theta}\right)$. Notice that $p_{l}-c_{H}<0$; that is, trading at the low price in state $H$ is worse than not trading at all.

Buyers discount the future with $0<\delta<1$; if one of them trades after $t$ periods and pays $p_{i}, i=l, h$, then his payoff is either $\delta^{t}\left(B-p_{i}\right)$ or $\delta^{t}\left(b-p_{i}\right)$ depending on his type. ${ }^{4}$

Formally, this problem is a multi-stage game with observed actions and incomplete information where Nature chooses $\theta=H$ with probability $\mu_{0}$. Then, if the state is $H(L)$, it randomly assigns the valuation $B$ to a proportion $\alpha(\epsilon)$ of the buyers and $b$ to the rest. Each buyer observes the state of the world and his valuation for the good; the seller does not observe these moves by Nature, but she is fully aware of the total number of buyers and their proportions in both states. After this, the game proceeds as described above; that is, in every period, each buyer meets the seller with probability $\frac{1}{M}$, the seller posts a price, the buyer accepts or rejects and if he rejects, the matching process starts again.

A behavior strategy for the seller specifies a probability distribution over prices for each nonterminal history at which she has to make a decision; $\sigma\left(p_{i} \mid h^{t}\right)$ is the probability that she announces $p_{i}, i=l, h$, given $h^{t}$. Notice that the only nonterminal $t$-history for the seller is of the form $h^{t}=$ $\left(\left(p_{h}, R\right)_{1},\left(p_{h}, R\right)_{2}, \ldots,\left(p_{h}, R\right)_{t}\right)$, since acceptance by the buyer ends the game. We denote by $\mu_{t}$ the probability the seller assigns to $H$ after the history $h^{t}$.

Similarly, a behavior strategy for a buyer maps nonterminal histories (at which he is matched with a seller) and types into probability distributions over actions. Formally, we can represent this strategy as $\left(\sigma\left(a \mid\left(h^{t}, p_{i}\right), H, B\right)\right.$, $\left.\sigma\left(a \mid\left(h^{t}, p_{i}\right), H, b\right), \sigma\left(a \mid\left(h^{t}, p_{i}\right), L, B\right), \sigma\left(a \mid\left(h^{t}, p_{i}\right), L, b\right)\right), a=A, R, i=h, l$; for example, $\sigma\left(A \mid\left(h^{t}, p_{h}\right), H, B\right)$ is the probability that a buyer accepts in period $t$, given that the price posted is $p_{h}$, the state is $H$ and his valuation is $B$.

The solution concept we use is Perfect Bayesian Equilibrium (PBE); that is, we look for a profile of behavior strategies (one for each player) and beliefs for the seller such that, at any stage of the game, strategies are optimal given the beliefs, and these are derived using Bayes' rule whenever possible.

Notice that we can immediately pin down the values of some of the components of a buyer's optimal strategy: since $p_{l}$ is always accepted, it follows that $\sigma\left(A \mid\left(h^{t}, p_{l}\right), H, B\right), \sigma\left(A \mid\left(h^{t}, p_{l}\right), H, b\right), \sigma\left(A \mid\left(h^{t}, p_{l}\right), L, B\right)$, and $\sigma\left(A \mid\left(h^{t}, p_{l}\right), L, b\right)$ are equal to one for every $t$. Also, $b<p_{h}$ implies that $\sigma\left(A \mid\left(h^{t}, p_{h}\right), H, b\right)$ and $\sigma\left(A \mid\left(h^{t}, p_{h}\right), L, b\right)$ are equal to zero in every period.

\footnotetext{
${ }^{3}$ Like the other papers cited in the introduction, we take the selling mechanism as given; we refer the reader to Lazear (1986) for practical reasons that can make posted prices the 'preferred' mechanism for the seller.

${ }^{4}$ As will become clear below, having different discount factors for the seller and the buyers affords a neat and intuitive characterization of the set of equilibria in terms of cut-off values for $\beta$ and $\delta$.
} 
The only buyers that need not have a dominant strategy are the ones with valuation equal to $B$ when the price posted is $p_{h}$. As we shall see, their behavior will play a prominent role in the determination of the seller's optimal pricing policy.

\section{Main results}

The decision problem that a $B$-buyer faces when the price posted in period $t$ is $p_{h}$ is the following: if he accepts, his payoff is $\delta^{t}\left(B-p_{h}\right)$ and the game is over; if he rejects, there is a positive probability that he will meet the seller again in the future and, having updated her beliefs after failing to trade, he may buy the good at $p_{l}$. In other words, rejection has an option value, and a $B$-buyer must compare it with the payoff from accepting.

Notice that in equilibrium, a necessary condition for a $B$-buyer to reject a price $p_{h}$ in any period is

$$
B-p_{h} \leq \frac{1}{M} \delta\left(B-p_{l}\right)
$$

That is, it must be the case that the payoff from buying at a high price is less than the expected value of buying at a low price next period.

Let $\delta^{*}=\frac{M\left(B-p_{h}\right)}{B-p_{l}}$; then, the condition stated above is equivalent to $\delta \geq \delta^{*}$. If this condition is satisfied we will say that buyers are patient, in the restricted sense that a buyer may find it optimal to reject a high price and wait for a low price; similarly, buyers are defined as impatient if $\delta<\delta^{*}$, i.e. they never find it optimal to reject a high price. Notice that $\delta^{*} \geq 1$ if the number of buyers is sufficiently large; in this event, with a slight abuse of the terminology, we will say that $B$-buyers are always impatient. ${ }^{5}$

\subsection{Impatient buyers}

If $\delta<\delta^{*}$, then a $B$-buyer will always find accepting $p_{h}$ optimal in both states. In other words, $\sigma\left(A \mid\left(h^{t}, p_{h}\right), L, B\right)$ and $\sigma\left(A \mid\left(h^{t}, p_{h}\right), H, B\right)$ will be equal to one.

It remains to characterize the seller's optimal strategy. Since buyers' strategies are stationary and independent of the seller's strategy, her problem can be formulated in stochastic dynamic programming terms using her beliefs as a state variable.

Let $\mu$ be the seller's current beliefs; the value function of the seller's problem satisfies the following Bellman equation: ${ }^{6}$

$$
\begin{aligned}
V(\mu)= & \max \left\{\mu\left(p_{l}-c_{H}\right)+(1-\mu)\left(p_{l}-c_{L}\right),\left(\mu \alpha\left(p_{h}-c_{H}\right)\right.\right. \\
& \left.\left.+(1-\mu) \epsilon\left(p_{h}-c_{L}\right)\right)+(\mu(1-\alpha)+(1-\mu)(1-\epsilon)) \beta V\left(\mu^{\prime}\right)\right\},
\end{aligned}
$$

5 This case will become relevant in Section 3.4, where the limit properties of the model are analyzed.

${ }^{6}$ It is straightforward to show that the Principle of Optimality holds in this problem, and that the solution to the functional equation we characterize is the value function of the seller's problem. 
where

$$
\mu^{\prime}=\frac{\mu(1-\alpha)}{\mu(1-\alpha)+(1-\mu)(1-\epsilon)} .
$$

The interpretation of (1) is straightforward: if she announces $p_{l}$, she trades with probability one and this has an expected payoff equal to

$\mu\left(p_{l}-c_{H}\right)+(1-\mu)\left(p_{l}-c_{L}\right)$. If she announces $p_{h}$, then the following events can happen: a) with probability $\mu$ the state is $H$, in which case she trades with probability $\alpha$ and gets $p_{h}-c_{H}$, and she fails to trade with probability $(1-\alpha)$ and continues the search, which has an expected discounted payoff (under an optimal strategy) given by $\beta V\left(\mu^{\prime}\right)$; b) with probability $(1-\mu)$ the state is $L$ and either she trades with probability $\epsilon$ getting $p_{h}-c_{L}$, or she fails to trade with probability $(1-\epsilon)$ and continues the search, which has an optimal expected discounted payoff given by $\beta V\left(\mu^{\prime}\right)$. Rearranging terms yields the second expression inside the max operator.

The best possible scenario for the seller is that the state be low and she sell the good immediately at a high price, in which case she would get $p_{h}-c_{L}$. Moreover, the seller always has a 'default strategy' that yields a nonnegative expected payoff: namely, 'always announce $p_{h}$ '. Therefore, a strategy with a negative expected discounted payoff can never be optimal. These remarks show that $0 \leq V(\mu) \leq p_{h}-c_{L}$.

The following proposition characterizes the seller's value function:

Proposition 1 There is a unique continuous and convex function $V:[0,1] \longrightarrow$ $\left[0, p_{h}-c_{L}\right]$ that solves the Bellman equation (1). ${ }^{7}$

Proof. See the Appendix.

We now turn to the characterization of the seller's optimal policy. For notational simplicity, define the functions $f(\mu)$ and $g(\mu)$ as follows:

$$
\begin{gathered}
f(\mu)=\mu\left(p_{l}-c_{H}\right)+(1-\mu)\left(p_{l}-c_{L}\right), \\
g(\mu)=\left(\mu \alpha\left(p_{h}-c_{H}\right)+(1-\mu) \epsilon\left(p_{h}-c_{L}\right)\right)+ \\
(\mu(1-\alpha)+(1-\mu)(1-\epsilon)) \beta V\left(\frac{\mu(1-\alpha)}{\mu(1-\alpha)+(1-\mu)(1-\epsilon)}\right) .
\end{gathered}
$$

Although we focus on the case where the seller is uninformed $(0<\mu<1)$, it is instructive to start with the informed case. Suppose $\mu=1$; intuitively, if she knows that the state is $H$, then she will always announce $p_{h}$ since $p_{l}$ leads to trade for sure at a loss $p_{l}-c_{H}<0$. The situation is a bit more complex when the seller knows that the state is low $(\mu=0)$. If she announces $p_{l}$ she trades with probability one and obtains a surplus given by $p_{l}-c_{L}$. However, for any given

\footnotetext{
7 The convexity of the value function in Bayesian control problems is a standard result; see Nyarko (1994), Proposition 3.2 and the references cited therein. The proof contained in the Appendix is needed in order to show that the second term inside the max operator is convex, a property that plays a key role in the proof of Proposition 2 (iii).
} 
proportion of $B$-buyers in state $L$, if her discount factor is sufficiently high she might find it optimal to announce $p_{h}$ instead. Since beliefs will not change in this case, if she announces $p_{h}$ in any particular period, she will continue to do so forever. Finally, if she is uncertain about the state of the world that prevails $(0<\mu<1)$, then the optimal policy is to announce a high price so long as her belief about the high state is above a threshold level, and to switch to a low price the first period it falls below the threshold. However, the fact that there is only a finite number of prices available also makes the policy 'always $p_{h}$ ' optimal if the seller's discount factor is high enough.

Let $\beta^{*}=\frac{\left(p_{l}-c_{L}\right)-\epsilon\left(p_{h}-c_{L}\right)}{(1-\epsilon)\left(p_{l}-c_{L}\right)}$; the following proposition characterizes the optimal strategy for the seller.

Proposition 2 (i) If $\mu=1$, then the optimal policy for the seller is to announce $p_{h}$ in every period and the value of this policy is $\frac{\alpha}{1-(1-\alpha) \beta}\left(p_{h}-c_{H}\right)$.

(ii) If $\mu=0$, then the optimal policy for the seller is the following:

If $\beta \geq \beta^{*}$, then she announces $p_{h}$ in every period. The expected discounted payoff of this strategy is $\frac{\epsilon}{(1-(1-\epsilon) \beta)}\left(p_{h}-c_{L}\right){ }^{8}$

If $\beta<\beta^{*}$, then she always announces $p_{l}$. The expected discounted payoff of this strategy is $\left(p_{l}-c_{L}\right)$.

(iii) If $0<\mu<1$, then the optimal policy for the seller is the following:

If $\beta<\beta^{*}$, then she announces $p_{h}$ if $\mu \geq \mu^{*}$, and $p_{l}$ if $\mu<\mu^{*}$. The threshold $\mu^{*}$ is the unique solution to the equation

$$
f\left(\mu^{*}\right)-g\left(\mu^{*}\right)=0 \text {. }
$$

If $\beta \geq \beta^{*}$, then she announces $p_{h}$ in every period.

Proof. See the Appendix.

This completes the characterization of the equilibrium strategies. In summary, the PBE of the game is described as follows:

Proposition 3 (i) If $\beta<\beta^{*}$, then the following strategies and beliefs constitute the unique PBE of the game when $\delta<\delta^{*}$ :

Buyers: $\sigma\left(A \mid\left(h^{t}, p_{i}\right), \theta, B\right)=1$ for every $t, i=l, h, \theta=H, L$; $\sigma\left(A \mid\left(h^{t}, p_{l}\right), \theta, b\right)=1, \sigma\left(A \mid\left(h^{t}, p_{h}\right), \theta, b\right)=0$ for every $t, \theta=H, L$.

Seller: $\sigma\left(p_{h} \mid h^{t}\right)=1$ for every $t$ in which $\mu_{t} \geq \mu^{*}$, and zero otherwise.

Beliefs: $\mu_{t}=\frac{(1-\alpha)^{t} \mu_{0}}{\mu_{0}(1-\alpha)^{t}+(1-\epsilon)^{t}\left(1-\mu_{0}\right)}$ for every $t$.

(ii) if $\beta \geq \beta^{*}$, then buyers' strategies and beliefs as in $(i)$, with the seller's strategy $\sigma\left(p_{h} \mid h^{t}\right)=1$ for every $t$, constitute the unique PBE of the game when $\delta<\delta^{*}$.

A special case of the PBE described above is one in which $\alpha=1$ and $\epsilon=0$; i.e., in state $H$, all buyers are $B$-buyers, and in state $L$, none are $B$ buyers. Then, $\beta^{*}=1$ and only (i) of Proposition 3 is relevant; moreover, given

\footnotetext{
${ }^{8}$ Without loss of generality, we assume that when $\beta=\beta^{*}$ the seller chooses 'always $p_{h}$ ', although this is only one of the optimal policies.
} 
the strategy of the buyers, a rejection is a noiseless signal of the state and therefore a single announcement of $p_{h}$ is all that is needed when $\mu_{0} \geq \mu^{*}$. This resembles the outcome of the (steady-state) equilibrium E1 characterized by Serrano and Yosha (1993) in the 'large market' set up. An intuitive way to explain this similarity is the following: notice that $\delta<\delta^{*}$ can be manipulated and rewritten as $\frac{1}{M}<\frac{1}{M^{*}}$, where $M^{*}=\frac{\delta\left(B-p_{l}\right)}{B-p_{h}}$; therefore, the straightforward behavior of buyers in equilibrium can be interpreted as arising because their number is 'large', and hence there is a small probability of meeting the seller again in the future. Unlike Serrano and Yosha (1993), the uniqueness of the PBE when $\frac{1}{M}<\frac{1}{M^{*}}$ is due to the fact that there is only one seller and one time entry, for this 'kills' a $B$-buyer's option value of a rejection.

When $\alpha<1$, a rejection is only a noisy signal of the true state, and the seller's belief changes in a slower fashion. The role that this noise plays in the seller's optimal strategy (and therefore in the PBE of the game) is investigated next.

\subsection{The incentives to announce a high price}

In this section, we analyze how the seller's equilibrium behavior changes when her discount factor or the information structure of her problem (i.e., the proportions of $B$-buyers in each state) change. The main result we obtain is that her incentives to announce and to experiment with a high price are not necessarily monotonic in the informativeness of the signals (rejections) she observes.

Notice that the seller's equilibrium strategy is summarized by the threshold $\mu^{*}$, which is a function of the parameters of the model; in particular, it depends on $\alpha, \epsilon$ and $\beta$. The following proposition derives an explicit formula for $\mu^{*}$ that allows us to characterize its behavior as a function of those parameters.

Proposition 4 (i) If $\beta<\beta^{*}$, then $\mu^{*}$ is positive and equal to

$$
\frac{(1-\beta(1-\epsilon))\left(p_{l}-c_{L}\right)-\epsilon\left(p_{h}-c_{L}\right)}{(1-\beta(1-\epsilon))\left(p_{l}-c_{L}\right)-\epsilon\left(p_{h}-c_{L}\right)+\alpha\left(p_{h}-c_{H}\right)-(1-\beta(1-\alpha))\left(p_{l}-c_{H}\right)} .
$$

(ii) If $\beta<\beta^{*}$, then $\mu^{*}$ is strictly decreasing in $\beta, \alpha$, and $\epsilon$; moreover, for given values of $\alpha$ and $\epsilon, \mu^{*}$ converges to zero as $\beta$ converges to $\beta^{*}$.

Proof. See the Appendix.

The intuition underlying these results is the following: as $\beta$ increases, the seller becomes more patient and therefore more willing to announce $p_{h}$; this is reflected in a lower threshold $\mu^{*}$. On the other hand, an increase in $\alpha$ has three effects:

a) it increases the likelihood of making a sale in the current period,

b) it makes a rejection of $p_{h}$ more informative in Blackwell's sense, and 
c) it makes the future 'less important' (i.e., $(\mu(1-\alpha)+(1-\mu)(1-\epsilon)) \beta$ decreases).

Effects a) and b) make the seller more prone to announce $p_{h}$, while c) works in the opposite direction; Proposition 4 shows that the first two effects dominate. The same effects arise when $\epsilon$ increases, although the direction of b) is reversed; however, effect a) prevails, and the net result is a decrease in the threshold $\mu^{*}$. In other words, despite the fact that the informational content of a rejection of $p_{h}$ decreases, the seller becomes more inclined to post $p_{h}{ }^{9}{ }^{9}$

Using expression (2), it is easy to show that the seller's equilibrium behavior involves experimentation; if the seller were 'myopic', she would ignore the impact that an announcement of $p_{h}$ had on future profits, and would set a belief threshold given by

$$
\mu^{m}=\frac{\left(p_{l}-c_{L}\right)-\epsilon\left(p_{h}-c_{L}\right)}{\left(c_{H}-c_{L}\right)-\epsilon\left(p_{h}-c_{L}\right)+\alpha\left(p_{h}-c_{H}\right)} .
$$

Since $\mu^{*}<\mu^{m}$, the seller announces $p_{h}$ when $\mu \in\left(\mu^{*}, \mu^{m}\right)$, although $p_{l}$ is myopically optimal. ${ }^{10}$

An interesting question is whether the length of the interval $\left(\mu^{*}, \mu^{m}\right)$ increases if a rejection becomes more informative. The following example reveals that this is not necessarily the case:

Example 1: Let $\epsilon=0$; straightforward differentiation shows that

$$
\frac{\partial\left(\mu^{m}-\mu^{*}\right)}{\partial \alpha} \begin{cases}<0 & \text { if } \alpha>\alpha^{*} \\ \geq 0 & \text { if } \alpha \leq \alpha^{*},\end{cases}
$$

where

$$
\alpha^{*}=\left(\frac{(1-\beta)\left(c_{H}-c_{L}\right)^{2}}{\left(p_{h}-c_{H}\right)^{2}+\beta\left(c_{H}-p_{l}\right)\left(p_{h}-c_{H}\right)}\right)^{\frac{1}{2}} .
$$

The intuition is as follows: since both kinds of sellers, myopic and nonmyopic, take into account effect a), it is the interplay between effects b) and c) that determines the sign of $\frac{\partial\left(\mu^{m}-\mu^{*}\right)}{\partial \alpha}$; the example shows that b) dominates for low values of $\alpha$, and c) prevails for high values. In other words, for low values of $\alpha$, the informational gain from posting $p_{h}$ offsets the reduction in the option value due to effect $\mathrm{c}$ ); the opposite happens for high values of $\alpha$.

\footnotetext{
${ }^{9}$ As another example, consider the case where $\epsilon=(1-\alpha)$; it can be shown that an increase in $\alpha$, which leads to more informative observations, results in a lower $\mu^{*}$.

${ }^{10}$ Notice that a myopic seller not only ignores that posting $p_{h}$ and being rejected provides her with information about the state of the world, but she also ignores the fact that if she does not sell today, she can still sell in the future. In this sense, the model exhibits what Datta, Mirman, and Schlee (1998) call signal dependence; i.e., the current signal (rejection of $p_{h}$ ) has an additional effect on the future discounted payoff (option to sell in the future) besides changing the seller's beliefs about the state. The difference between $\mu^{m}$ and $\mu^{*}$ includes both effects; see the discussion after Example 1.
} 


\subsection{Patient buyers}

We now consider the case where $\delta \geq \delta^{*}$. In this situation, a $B$-buyer need not always accept $p_{h}$ : since a rejection today can have a positive option value, he may have incentives to turn down a high price hoping that the next time he meets the seller she will post a low price. This strategic behavior decreases the informational content of a rejection for the seller, and it therefore affects her optimal pricing strategy.

Notice that, if $\beta \geq \beta^{*}$, then the PBE described in Proposition 3 (ii) is still a PBE when buyers are patient. For if the seller announces a high price in every period, then a $B$-buyer cannot improve his payoff by rejecting it.

However, the strategies and beliefs given in Proposition 3 (i) do not constitute a PBE anymore. The explanation is simple: given the strategy of the seller, a $B$-buyer will reject $p_{h}$ if he meets the seller the period before her belief $\mu$ reaches $\mu^{*}$; thus, there is a nonterminal history after which $B$-buyers have a profitable deviation, so the profile of strategies and beliefs described in Proposition 3 (i) cannot be a PBE when $\delta \geq \delta^{*}$.

Let $\mu^{* *}$ be the belief that solves $\left(p_{l}-c_{L}\right)-\mu\left(c_{H}-c_{L}\right)=0$; i.e., $\mu^{* *}=$ $\frac{p_{l}-c_{L}}{c_{H}-c_{L}}$. This is the largest value of $\mu$ (the belief about $H$ ) that gives the seller a nonnegative expected payoff from trading at $p_{l}$. She would never announce $p_{l}$ if $\mu>\mu^{* *}$, for this would yield a negative expected payoff.

Proposition 5 If $\mu_{0} \leq \mu^{* *}$, then the following strategies and beliefs constitute a $P B E$ of the game when $\delta \geq \delta^{*}$ :

Buyers: $\sigma\left(A \mid\left(h^{t}, p_{l}\right), \theta, B\right)=1, \sigma\left(A \mid\left(h^{t}, p_{h}\right), \theta, B\right)=0, \sigma\left(A \mid\left(h^{t}, p_{l}\right), \theta, b\right)=$ 1 and $\sigma\left(A \mid\left(h^{t}, p_{h}\right), \theta, b\right)=0$ for all $t, \theta=H, L$.

Seller: $\sigma\left(p_{h} \mid h^{t}\right)=0$ for all $t$.

Beliefs: $\mu_{t}=\mu_{0}$ for all $t$.

Proof. Given the strategies of the buyers and the fact that $\mu_{0} \leq \mu^{* *}$, the optimal strategy for the seller is to announce $p_{l}$ after any nonterminal history. Similarly, given the seller's strategy, it is optimal for a $B$-buyer to reject $p_{h}$ after any nonterminal history, since $\delta \geq \delta^{*}$ makes it worthwhile for him to wait for a low price in the next period. The beliefs stated in the proposition support the optimality of these strategies in every period.

In other words, when $\delta \geq \delta^{*}$, there is an equilibrium in which $B$-buyers always reject a high price, and the seller's only alternative is to post a low price and sell in the first period; in order for this to be optimal for her, it must be the case that selling at a low price yields a nonnegative expected payoff, and this is true so long as $\mu_{0} \leq \mu^{* *}$. Notice that, compared to the case with $\delta<\delta^{*}$, the strategic behavior of $B$-buyers induces the seller to announce a low price when $\mu_{0} \in\left(\mu^{*}, \mu^{* *}\right)$, values that previously made her post $p_{h}$.

If $\mu_{0}>\mu^{* *}$, then it is easy to see that 'always $p_{l}$ ' cannot be an optimal strategy for the seller; so long as $\mu_{t}>\mu^{* *}$, announcing $p_{h}$ and being rejected is strictly better than posting $p_{l}$ (which has a negative expected payoff). Therefore, if $\mu_{0}>\mu^{* *}$, then in any PBE the seller's strategy must include announcements 
of $p_{h}$ at least until the first period in which her belief about the high state drops below $\mu^{* *}$. Also, notice that if the seller is expected to switch to $p_{l}$ at any particular $t$, then a $B$-buyer will reject $p_{h}$ at $t-1$; therefore, the seller is better off by switching at $t-1$ instead of at $t$. This suggests that any PBE must involve randomization after a certain point in time.

The following example, which is derived in the appendix, illustrates these features. To simplify the notation, set $\sigma\left(A \mid\left(h^{t}, p_{h}\right), H, B\right)=\pi_{t}$, and define $\hat{t}$ as the first time that $\mu_{t}$ drops below $\mu^{* *}$.

Example 2: Let $\alpha=\frac{1}{M}, \epsilon=0$, and $\mu_{0}>\mu^{* *}$. Then there is a PBE with the following characteristics:

(i) the seller announces $p_{h}$ until $\hat{t}$, and then posts $p_{h}$ with probability $0<$ $\lambda<1$ forever after,

(ii) the $B$-buyer accepts $p_{h}$ if he meets the seller before $\hat{t}$, and then randomizes between acceptance and rejection with probability $0<\pi_{t}<1$,

(iii) $\pi_{t}$ increases over time and converges to one as $\mu_{t}$ converges to $\mu^{*}$.

If $\mu^{*}<\mu_{0}<\mu^{* *}$, then (i)-(iii) hold with $\hat{t}=0$.

In this example, the seller announces $p_{h}$ until $p_{l}$ ceases to have a negative expected payoff, and then she randomizes from that point onward. The $B$-buyer accepts $p_{h}$ with probability one if he meets the seller before $\hat{t}$; after that, she randomizes between acceptance and rejection of a high price with probabilities that change over time in such a way that they keep the seller indifferent between $p_{l}$ and $p_{h}$ in every period. In order to accomplish this, the probability that he accepts $p_{h}$ increases over time; the fact that $p_{h}$ is more likely to be accepted in the future provides the seller with incentives to post $p_{h}$ with positive probability in every period.

\subsection{Limit properties}

So far we have studied the equilibria of the game for fixed discount factors $\beta, \delta \in[0,1)$. We now investigate what happens when both discount factors tend to one; i.e., when the cost of waiting becomes negligible.

Our main result is that, although it is easy to construct PBE where the seller trades at the 'wrong' price $\left(p_{l}\right.$ in state $\left.H\right)$, there are PBE in the limit where trade always occurs at ex-post individually rational prices. This contrasts with the large market model with one time entry, analyzed by Blouin and Serrano (1998), where in all equilibria there is a nonnegligible fraction of agents that trades at the wrong price in the limit. ${ }^{11}$

An example of a PBE in which the seller trades at the wrong price in the limit is the following:

\footnotetext{
11 The steady-state version studied in Serrano and Yosha (1993) doesn't exhibit this feature: among other results, the authors show that there are always equilibria where trade occurs at the 'right' price.
} 
Proposition 6 If $\mu_{0}<\mu^{* *}$, then the PBE described in Proposition 5 is an equilibrium in the limit, as $\delta \rightarrow 1$ and $\beta \rightarrow 1$.

Proof. See the Appendix.

The outcome of this PBE is the following: the seller announces $p_{l}$ in the first period and trades. Thus, trade occurs at the wrong price if the state happens to be $H$.

Our model also has PBE where trade occurs at the 'right price' in the limit:

Proposition 7 For any $\mu_{0}$ :

(i) The PBE described in Proposition 3 (ii) is an equilibrium in the limit, as $\delta \rightarrow 1$ and $\beta \rightarrow 1$.

(ii) If $\alpha=1, \epsilon=0$, and the primitives of the model are such that $\delta^{*} \geq 1$, then the PBE described in Proposition 3 (i) is an equilibrium in the limit, as $\delta \rightarrow 1$ and $\beta \rightarrow 1$.

Proof. See the Appendix.

Let us interpret the PBE described in Propositions 6 and 7 in terms of the following two forces identified by Serrano and Yosha (1993) as the discount factors tend to one:

CL (cost of learning): as the seller becomes more patient, it is less costly for her to sample buyers in order to acquire information about the state of the world;

MI (misrepresentation of information): as $B$-buyers become more patient, it is less costly for them to reject $p_{h}$ (the option value of a rejection increases).

Consider the PBE described in Proposition 6, in which the seller posts $p_{l}$ in every period and trades in period one. Since in this case posting $p_{l}$ has a nonnegative expected payoff for the seller, this gives $B$-buyers incentives to reject $p_{h}$ in every period; in other words, MI prevails and no learning takes place.

In the PBE described in Proposition 7 (i), the seller never trades at the wrong price; that is, she never sells the good at $p_{l}$ in state $H$. The intuition is the following: no matter what the true state is, it is optimal for the seller to post $p_{h}$ in every period; in other words, CL is zero. This makes MI too costly for $B$-buyers, inducing them to behave truthfully.

Finally, in the PBE described in Proposition 7 (ii) with $\alpha=1, \epsilon=0$, and $\delta^{*} \geq 1$, MI is not active, and CL goes to zero as $\beta \rightarrow 1$; this makes $p_{h}$ optimal in the first period no matter what the seller's belief is, and a rejection reveals that the state is $L$ with probability one. ${ }^{12}$ Thus, she either trades in the first period at $p_{h}$ if the state is $H$, or in the second period at $p_{l}$ if the state is $L$.

Intuitively, we can explain the difference between our results and those of Blouin and Serrano (1998) as follows: unlike their model, ours allows for heterogeneity of buyers' valuations in each state of the world, and we drop the assumption of equal number of buyers and sellers. The presence of $B$ and $b$-buyers

\footnotetext{
${ }^{12}$ Recall that, in this case, $\beta^{*}=1$, and $\mu^{*}$ converges to zero as $\beta \rightarrow 1$ (Proposition 4 (ii)).
} 
in each state allows the seller to commit herself to post $p_{h}$ in every period until someone accepts. This cannot happen without heterogeneity in each state, because one rejection reveals $L$ with certainty when buyers behave truthfully, and it is optimal for the seller to post $p_{l}$ from that point onward; in other words, she can only make a commitment to post $p_{h}$ for one period, and patient $B$-buyers will behave truthfully in the limit only if $\delta^{*} \geq 1$. Notice that $\delta^{*} \geq 1$ if $M$, the number of buyers, is sufficiently large; in other words, dropping Blouin and Serrano's assumption of equal number of buyers and sellers makes an important difference.

\section{Concluding remarks}

We have analyzed an infinite horizon single-sale game between a seller and a population of buyers. In this set-up with one time entry, asymmetric information, heterogeneous buyers, and active learning, we have shown that the seller's incentives to post a high price and to experiment are not necessarily monotonic in the informativeness of the signals she observes. Moreover, we have demonstrated that there exist PBE with the property that, as the discount factors tend to one, the seller always trades at an ex-post individually rational price in the limit.

It would be interesting to investigate whether these results hold in more general optimal stopping problems with learning and in a large market set-up like Wolinsky's model, when agents have heterogeneous valuations and the number of buyers is not equal to the number of sellers.

\section{Appendix}

Proof of Proposition 1. Let $C([0,1])$ be the space of continuous functions $h$ : $[0,1] \longrightarrow \mathbb{R}$, endowed with the sup norm. It is well known that this is a Banach space.

Define the operator $T$ as

$$
\begin{aligned}
\operatorname{Th}(\mu)= & \max \left\{\mu\left(p_{l}-c_{H}\right)+(1-\mu)\left(p_{l}-c_{L}\right),\left(\mu \alpha\left(p_{h}-c_{H}\right)\right.\right. \\
& \left.\left.+(1-\mu) \epsilon\left(p_{h}-c_{L}\right)\right)+(\mu(1-\alpha)+(1-\mu)(1-\epsilon)) \beta h\left(\mu^{\prime}\right)\right\},
\end{aligned}
$$

where

$$
\mu^{\prime}=\frac{\mu(1-\alpha)}{\mu(1-\alpha)+(1-\mu)(1-\epsilon)} .
$$

Since the two terms inside the max operator are continuous, $T$ maps $C([0,1])$ into itself; i.e., $T: C([0,1]) \longrightarrow C([0,1])$. Moreover, $T$ satisfies Blackwell sufficient conditions for a contraction:

monotonicity: Suppose that $h$ and $j$ are two elements in $C([0,1])$ such that $h(\mu) \leq j(\mu), \forall \mu \in[0,1]$. Then, 


$$
\begin{aligned}
\operatorname{Th}(\mu)= & \max \left\{\mu\left(p_{l}-c_{H}\right)+(1-\mu)\left(p_{l}-c_{L}\right),\left(\mu \alpha\left(p_{h}-c_{H}\right)\right.\right. \\
& \left.\left.+(1-\mu) \epsilon\left(p_{h}-c_{L}\right)\right)+(\mu(1-\alpha)+(1-\mu)(1-\epsilon)) \beta h\left(\mu^{\prime}\right)\right\} \\
\leq & \max \left\{\mu\left(p_{l}-c_{H}\right)+(1-\mu)\left(p_{l}-c_{L}\right),\left(\mu \alpha\left(p_{h}-c_{H}\right)\right.\right. \\
& \left.\left.+(1-\mu) \epsilon\left(p_{h}-c_{L}\right)\right)+(\mu(1-\alpha)+(1-\mu)(1-\epsilon)) \beta j\left(\mu^{\prime}\right)\right\} \\
= & T j(\mu) ;
\end{aligned}
$$

discounting: Let $a$ be a nonnegative constant. Then,

$$
\begin{gathered}
T(h(\mu)+a)=\max \left\{\mu\left(p_{l}-c_{H}\right)+(1-\mu)\left(p_{l}-c_{L}\right),\left(\mu \alpha\left(p_{h}-c_{H}\right)+(1-\mu)\right.\right. \\
\left.\left.\epsilon\left(p_{h}-c_{L}\right)\right)+(\mu(1-\alpha)+(1-\mu)(1-\epsilon)) \beta\left(h\left(\mu^{\prime}\right)+a\right)\right\} \\
\leq \max \left\{\mu\left(p_{l}-c_{H}\right)+(1-\mu)\left(p_{l}-c_{L}\right),\left(\mu \alpha\left(p_{h}-c_{H}\right)+(1-\mu)\right.\right. \\
\left.\left.\epsilon\left(p_{h}-c_{L}\right)\right)+(\mu(1-\alpha)+(1-\mu)(1-\epsilon)) \beta h\left(\mu^{\prime}\right)+\beta a\right\} \\
\leq \max \left\{\mu\left(p_{l}-c_{H}\right)+(1-\mu)\left(p_{l}-c_{L}\right),\left(\mu \alpha\left(p_{h}-c_{H}\right)+(1-\mu)\right.\right. \\
\left.\left.\epsilon\left(p_{h}-c_{L}\right)\right)+(\mu(1-\alpha)+(1-\mu)(1-\epsilon)) \beta h\left(\mu^{\prime}\right)\right\}+\beta a \\
=\operatorname{Th}(\mu)+\beta a .
\end{gathered}
$$

Therefore, $T$ is a contraction and, by the Contraction Mapping Theorem, there is a unique $V \in C([0,1])$ such that $T V=V$.

Let $C^{\prime}([0,1])$ be the space of functions in $C([0,1])$ that are also convex. It is straightforward to show that $C^{\prime}([0,1])$ is a closed subset of $C([0,1])$. Therefore, in order to show that $V$ is convex, it is enough to prove that $T: C^{\prime}([0,1]) \longrightarrow$ $C^{\prime}([0,1])$.

Let $h(\mu)$ be an element of $C^{\prime}([0,1])$, and consider two different beliefs $\mu_{1}$ and $\mu_{2}$ and a scalar $\lambda \in[0,1]$. We must show that

$$
\operatorname{Th}\left(\lambda \mu_{1}+(1-\lambda) \mu_{2}\right) \leq \lambda \operatorname{Th}\left(\mu_{1}\right)+(1-\lambda) \operatorname{Th}\left(\mu_{2}\right) .
$$

Notice that $T h$ is the maximum of two functions. If we can show that these functions are convex, then since the maximum of two convex functions is convex, $T h$ will inherit the convexity of $h$. The first term inside the max operator is linear and therefore convex. Call this term $f(\mu)$.

Consider the second term. For notational simplicity, define $\mu_{c}=\lambda \mu_{1}+(1-$ 入) $\mu_{2}$. At $\mu_{c}$, the second term inside the max operator is

$$
\begin{aligned}
g\left(\mu_{c}\right)= & \left(\mu_{c} \alpha\left(p_{h}-c_{H}\right)+\left(1-\mu_{c}\right) \epsilon\left(p_{h}-c_{L}\right)\right) \\
& +\left(\mu_{c}(1-\alpha)+\left(1-\mu_{c}\right)(1-\epsilon)\right) \beta h\left(\mu_{c}^{\prime}\right),
\end{aligned}
$$

where

$$
\mu_{c}^{\prime}=\frac{\left(\lambda \mu_{1}+(1-\lambda) \mu_{2}\right)(1-\alpha)}{\left(\lambda \mu_{1}+(1-\lambda) \mu_{2}\right)(1-\alpha)+\left(1-\left(\lambda \mu_{1}+(1-\lambda) \mu_{2}\right)\right)(1-\epsilon)} .
$$

After some manipulation, $\mu_{c}^{\prime}$ can be written as $\gamma \mu_{1}^{\prime}+(1-\gamma) \mu_{2}^{\prime}$, where $\gamma=\frac{\lambda\left(\mu_{1}(1-\alpha)+\left(1-\mu_{1}\right)(1-\epsilon)\right)}{\lambda\left(\mu_{1}(1-\alpha)+\left(1-\mu_{1}\right)(1-\epsilon)\right)+(1-\lambda)\left(\mu_{2}(1-\alpha)+\left(1-\mu_{2}\right)(1-\epsilon)\right)}$. 
Since $h\left(\mu_{c}^{\prime}\right)$ is convex,

$$
h\left(\mu_{c}^{\prime}\right)=h\left(\gamma \mu_{1}^{\prime}+(1-\gamma) \mu_{2}^{\prime}\right) \leq \gamma h\left(\mu_{1}^{\prime}\right)+(1-\gamma) h\left(\mu_{2}^{\prime}\right) .
$$

Using this, it is easy to show that

$$
g\left(\mu_{c}\right) \leq \lambda g\left(\mu_{1}\right)+(1-\lambda) g\left(\mu_{2}\right) .
$$

which proves that $g$ is convex. Since the maximum of two convex functions is also convex, it follows that $\operatorname{Th}(\mu)=\max \{f(\mu), g(\mu)\}$ is convex, and hence the fixed point of $T$ is convex, too. ${ }^{13}$

Proof of Proposition 2. (i) Notice that $\mu=1$ implies that $\mu^{\prime}=1$. The Bellman equation becomes,

$$
V(1)=\max \left\{p_{l}-c_{H}, \alpha\left(p_{h}-c_{H}\right)+(1-\alpha) \beta V(1)\right\} .
$$

The first term inside the max operator is negative, while the second is a convex combination of nonnegative terms. Therefore, the optimal strategy is to announce $p_{h}$ in every period and the expected payoff is the solution to

$$
V(1)=\alpha\left(p_{h}-c_{H}\right)+(1-\alpha) \beta V(1),
$$

which rearranges to

$$
V(1)=\frac{\alpha}{1-(1-\alpha) \beta}\left(p_{h}-c_{H}\right),
$$

completing the proof of part (i).

(ii) If $\mu=0$, then $\mu^{\prime}=0$. The Bellman equation becomes

$$
V(0)=\max \left\{p_{l}-c_{L}, \epsilon\left(p_{h}-c_{L}\right)+(1-\epsilon) \beta V(0)\right\} .
$$

The seller faces the same problem in every period. Consequently, she will always announce $p_{h}$ or she will always announce $p_{l}$. In the first case, the expected discounted payoff is the solution to

$$
J(0)=\epsilon\left(p_{h}-c_{L}\right)+(1-\epsilon) \beta J(0)
$$

which rearranges

$$
J(0)=\frac{\epsilon}{(1-(1-\epsilon) \beta)}\left(p_{h}-c_{L}\right) .
$$

If she finds it optimal to announce $p_{l}$ in every period, then the value of this policy is $p_{l}-c_{L}$.

\footnotetext{
${ }^{13}$ Convexity implies that $V(\cdot)$ is a.e. differentiable (Royden (1988), Proposition 17, pp.113-114). We note in passing that the only place where the value function is not differentiable is at the intersection of $f(\cdot)$ and $g(\cdot)$, where it has a kink.
} 
Announcing $p_{h}$ in every period is optimal if

$$
\beta>\frac{\left(p_{l}-c_{L}\right)-\epsilon\left(p_{h}-c_{L}\right)}{(1-\epsilon)\left(p_{l}-c_{L}\right)} .
$$

If this does not hold, then always $p_{l}$ ' is optimal.

(iii) Consider first the case where $\beta<\frac{\left(p_{l}-c_{L}\right)-\epsilon\left(p_{h}-c_{L}\right)}{(1-\epsilon)\left(p_{l}-c_{L}\right)}$, and define the function $D(\mu)=g(\mu)-f(\mu)$. It follows from (i) and (ii) that $D(1)>0$ and $D(0)<0$. Moreover, it follows from Proposition 1 that $D(\mu)$ is continuous and convex for $0 \leq \mu \leq 1$. The first three properties of $H$ ensure that there exists at least one $\mu^{*}$ such that $D\left(\mu^{*}\right)=0$. We now prove that $\mu^{*}$ is unique. Without loss of generality, suppose that $\mu_{a}^{*}$ and $\mu_{b}^{*}, \mu_{a}^{*}<\mu_{b}^{*}$, are such that $D\left(\mu_{a}^{*}\right)=D\left(\mu_{b}^{*}\right)=0$. Since $D$ is convex, it must be true that for any $\lambda \in(0,1)$ and $0 \leq \mu, \mu " \leq 1$,

$$
D\left(\lambda \mu+(1-\lambda) \mu^{\prime \prime}\right) \leq \lambda D(\mu)+(1-\lambda) D\left(\mu^{\prime \prime}\right)
$$

In particular, this must be true for $\lambda=\frac{\mu_{a}^{*}}{\mu_{b}^{*}}, \mu=\mu_{b}^{*}$ and $\mu "=0$. However,

$$
D\left(\frac{\mu_{a}^{*}}{\mu_{b}^{*}} \mu_{b}^{*}+\left(1-\frac{\mu_{a}^{*}}{\mu_{b}^{*}}\right) 0\right)=D\left(\mu_{a}^{*}\right)=0
$$

and

$$
\frac{\mu_{a}^{*}}{\mu_{b}^{*}} D\left(\mu_{b}^{*}\right)+\left(1-\frac{\mu_{a}^{*}}{\mu_{b}^{*}}\right) D(0)=\left(1-\frac{\mu_{a}^{*}}{\mu_{b}^{*}}\right) D(0)<0 .
$$

Therefore,

$$
D\left(\frac{\mu_{a}^{*}}{\mu_{b}^{*}} \mu_{b}^{*}+\left(1-\frac{\mu_{a}^{*}}{\mu_{b}^{*}}\right) 0\right)>\frac{\mu_{a}^{*}}{\mu_{b}^{*}} D\left(\mu_{b}^{*}\right)+\left(1-\frac{\mu_{a}^{*}}{\mu_{b}^{*}}\right) D(0),
$$

contradicting the convexity of $D$. Hence, $\mu^{*}$ is unique.

Since $f<g$ for $\mu>\mu^{*}$, and $f>g$ for $\mu<\mu^{*}$, it is optimal for the seller to announce $p_{h}$ if $\mu>\mu^{*}$ and $p_{l}$ if $\mu \leq \mu^{*}$.

Consider now the case where $\beta \geq \frac{\left(p_{l}-c_{L}\right)-\epsilon\left(p_{h}-c_{L}\right)}{(1-\epsilon)\left(p_{l}-c_{L}\right)}$. It follows from (i) and (ii) that $D(1)>0$ and $D(0)>0$. Since $D(\cdot)$ is convex, the right derivative exists at each point in its domain (Royden (1988), Proposition 17, pp.113-114.). If we can show that the right derivative $D_{+}^{\prime}(0)$ is positive, then it will follow that $D(\mu)>0$, $\forall \mu \in[0,1]$; otherwise, convexity would be violated. But $D(\mu)>0, \forall \mu \in[0,1]$ is equivalent to $g(\mu)>f(\mu) \forall \mu \in[0,1]$; in words, this says that always $p_{h}$ ' is optimal.

The continuity of $D(\cdot)$ and the fact that $D(0)>0$ imply that $g(\cdot)$ is greater than $f(\cdot)$ in a right neighborhood of the origin. Hence $V(\mu)=g(\mu)$ for $\mu$ near 0 ; since $\mu^{\prime}<\mu, V\left(\mu^{\prime}\right)=g\left(\mu^{\prime}\right)$ also. Since $V(\cdot)$ and $g(\cdot)$ are convex, their right derivatives at 0 exist; moreover $V_{+}^{\prime}(0)=g_{+}^{\prime}(0)$. Thus, 


$$
\begin{aligned}
g_{+}^{\prime}(0)= & \alpha\left(p_{h}-c_{H}\right)-\epsilon\left(p_{h}-c_{L}\right)+((1-\alpha)-(1-\epsilon)) \beta V(0) \\
& +(1-\epsilon) \beta V_{+}^{\prime}(0) \frac{d \mu^{\prime}}{d \mu}(0) \\
= & \alpha\left(p_{h}-c_{H}\right)-\epsilon\left(p_{h}-c_{L}\right)+(\epsilon-\alpha) \beta \frac{\epsilon\left(p_{h}-c_{L}\right)}{1-(1-\epsilon) \beta} \\
& +(1-\epsilon) \beta g_{+}^{\prime}(0) \frac{1-\alpha}{1-\epsilon} .
\end{aligned}
$$

After some manipulation, this rearranges to

$$
g_{+}^{\prime}(0)=\frac{(\alpha-\epsilon)(1-\beta)}{(1-\beta(1-\alpha))(1-\beta(1-\epsilon))} p_{h}-\frac{\alpha}{1-\beta(1-\alpha)} c_{H}+\frac{\epsilon}{1-\beta(1-\epsilon)} c_{L} .
$$

Since $f^{\prime}(0)=c_{L}-c_{H}$, it follows that $D_{+}^{\prime}(0)=g_{+}^{\prime}(0)-f^{\prime}(0)>0$ if and only if

$$
\begin{aligned}
\frac{(\alpha-\epsilon)(1-\beta)}{(1-\beta(1-\alpha))(1-\beta(1-\epsilon))} p_{h} & +\left(1-\frac{\alpha}{1-\beta(1-\alpha)}\right) c_{H} \\
+ & \left(\frac{\epsilon}{1-\beta(1-\epsilon)}-1\right) c_{L}>0
\end{aligned}
$$

which is equivalent to

$$
\frac{(\alpha-\epsilon)(1-\beta)}{(1-\beta(1-\alpha))(1-\beta(1-\epsilon))} p_{h}+\frac{(1-\alpha)(1-\beta)}{1-\beta(1-\alpha)} c_{H}-\frac{(1-\epsilon)(1-\beta)}{1-\beta(1-\epsilon)} c_{L}>0 .
$$

Since $p_{h}>c_{H}>c_{L}$ and the coefficients in $p_{h}$ and $c_{H}$ are positive because $\alpha>\epsilon$ and $0<\alpha, \epsilon, \beta<1$,

$$
\begin{gathered}
\frac{(\alpha-\epsilon)(1-\beta)}{(1-\beta(1-\alpha))(1-\beta(1-\epsilon))} p_{h}+\frac{(1-\alpha)(1-\beta)}{1-\beta(1-\alpha)} c_{H}-\frac{(1-\epsilon)(1-\beta)}{1-\beta(1-\epsilon)} c_{L}> \\
\left(\frac{(\alpha-\epsilon)(1-\beta)}{(1-\beta(1-\alpha))(1-\beta(1-\epsilon))}+\frac{(1-\alpha)(1-\beta)}{1-\beta(1-\alpha)}-\frac{(1-\epsilon)(1-\beta)}{1-\beta(1-\epsilon)}\right) c_{L}=0 .
\end{gathered}
$$

Hence, $D_{+}^{\prime}(0)>0$ and the proof is complete.

Proof of Proposition 4. It is easy to show that the restriction on $\beta$ implies that $\mu^{*}$ is positive. From Proposition 2 (ii), this restriction also implies that $V(0)=f(0)>$ $g(0)$. Since $f$ and $g$ are continuous, $V(\mu)=f(\mu)$ in some right neighborhood of 0 . But $\alpha>\epsilon$ and $\mu^{\prime} \leq \mu$ implies that $V\left(\mu^{\prime}\right)=f\left(\mu^{\prime}\right)$ for $\mu$ in that right neighborhood. In fact, we have $V(\mu)=f(\mu)$ and $V\left(\mu^{\prime}\right)=f\left(\mu^{\prime}\right)$ as long as $0 \leq \mu \leq \mu^{*}$. Therefore, when $0 \leq \mu \leq \mu^{*}$ the function $g(\mu)$ is given by

$$
\begin{aligned}
g(\mu)= & \mu \alpha\left(p_{h}-c_{H}\right)+(1-\mu) \epsilon\left(p_{h}-c_{L}\right) \\
& +(\mu(1-\alpha)+(1-\mu)(1-\epsilon)) \beta f\left(\mu^{\prime}\right) \\
= & \mu \alpha\left(p_{h}-c_{H}\right)+(1-\mu) \epsilon\left(p_{h}-c_{L}\right) \\
& +\beta\left(\mu(1-\alpha)\left(p_{l}-c_{H}\right)+(1-\mu)(1-\epsilon)\left(p_{l}-c_{L}\right)\right) .
\end{aligned}
$$

This equation and the fact that $\mu^{*}$ is defined as the solution to $f\left(\mu^{*}\right)=g\left(\mu^{*}\right)$ allow us to obtain, after some manipulation, expression (2). 
Straightforward differentiation of $\mu^{*}$ confirms that $\frac{\partial \mu^{*}}{\partial \beta}<0, \frac{\partial \mu^{*}}{\partial \alpha}<0$, and $\frac{\partial \mu^{*}}{\partial \epsilon}<0$. of (2).

That $\mu^{*}$ converges to zero as $\beta \longrightarrow \frac{\left(p_{l}-c_{L}\right)-\epsilon\left(p_{h}-c_{L}\right)}{(1-\epsilon)\left(p_{l}-c_{L}\right)}$ is obvious by inspection

Derivation of the PBE of Example 2. The optimality of the strategies and beliefs up to period $\hat{t}$ is obvious, as well as the behavior of $b$-buyers in every $t$. Therefore, we shall focus on what happens after $\hat{t}$.

For the B-buyer to find it optimal to randomize between accepting and rejecting a high price, it must be the case that the seller's behavior strategy $\lambda$ leaves him indifferent between accept $p_{h}$ today', which gives him $B-p_{h}$, and reject $p_{h}$ today but accept it in the next meeting with the seller', which yields an expected payoff

$$
\frac{\delta}{M-\delta(M-1) \lambda}\left(\lambda\left(B-p_{h}\right)+(1-\lambda)\left(B-p_{l}\right)\right) .
$$

In other words, $\lambda$ must satisfy

$$
B-p_{h}=\frac{\delta}{M-\delta(M-1) \lambda}\left(\lambda\left(B-p_{h}\right)+(1-\lambda)\left(B-p_{l}\right)\right),
$$

which yields

$$
\lambda=\frac{\delta\left(B-p_{l}\right)-M\left(B-p_{h}\right)}{\delta\left(B-p_{l}\right)-\delta M\left(B-p_{h}\right)} .
$$

Since $\delta \geq \delta^{*}$ and $0<\delta<1$, it follows that $0<\lambda<1$. Therefore, our candidate for the seller's equilibrium strategy is $\sigma\left(p_{h} \mid h^{t}\right)=\lambda$ for all $t \geq \hat{t}$.

Now, a necessary condition for the seller to randomize between $p_{l}$ and $p_{h}$ in every period when the $B$-buyer accepts $p_{h}$ at any $t \geq \hat{t}$ with probability $\pi_{t}$, is that she be indifferent between 'announce $p_{l}$ today' and announce $p_{h}$ today and $p_{l}$ tomorrow'. The first choice yields

$$
f\left(\mu_{t}\right)=p_{l}-c_{L}-\mu_{t}\left(c_{H}-c_{L}\right),
$$

while the second has an expected payoff given by

$$
\begin{aligned}
\mu_{t}\left(\alpha \left(\pi_{t}\left(p_{h}-c_{H}\right)+\right.\right. & \left.\left(1-\pi_{t}\right) \beta\left(\mu_{t+1}\left(p_{l}-c_{H}\right)+\left(1-\mu_{t+1}\right)\left(p_{l}-c_{L}\right)\right)\right) \\
& \left.+(1-\alpha) \beta\left(\mu_{t+1}\left(p_{l}-c_{H}\right)+\left(1-\mu_{t+1}\right)\left(p_{l}-c_{L}\right)\right)\right) \\
& \left.+\left(1-\mu_{t}\right) \beta\left(\mu_{t+1}\left(p_{l}-c_{H}\right)+\left(1-\mu_{t+1}\right)\left(p_{l}-c_{L}\right)\right)\right),
\end{aligned}
$$

where

$$
\mu_{t+1}=\frac{\left((1-\alpha)+\alpha\left(1-\pi_{t}\right)\right) \mu_{t}}{\mu_{t}\left((1-\alpha)+\alpha\left(1-\pi_{t}\right)\right)+\left(1-\mu_{t}\right)} .
$$

Plugging $\mu_{t+1}$ in (3) gives, after some manipulation

$$
z\left(\pi_{t}, \mu_{t}\right)=\pi_{t} a\left(\mu_{t}\right)+\beta f\left(\mu_{t}\right)
$$


where

$$
a\left(\mu_{t}\right)=\alpha \mu_{t}\left(p_{h}-c_{H}+\beta\left(c_{H}-p_{l}\right)\right)>0 .
$$

Notice that $z\left(0, \mu_{t}\right)<f\left(\mu_{t}\right), \frac{\partial z}{\partial \pi_{t}}>0$ and, if $\mu_{t}>\mu^{*}$, then $z\left(1, \mu_{t}\right)>f\left(\mu_{t}\right)$. Thus, there is a unique $0<\pi_{t}\left(\mu_{t}\right)<1$ that solves $z\left(\pi_{t}, \mu_{t}\right)=f\left(\mu_{t}\right)$, given by

$$
\pi_{t}=\frac{(1-\beta)\left(p_{l}-c_{L}-\mu_{t}\left(c_{H}-c_{l}\right)\right)}{\alpha \mu_{t}\left(p_{h}-c_{H}+\beta\left(c_{H}-p_{l}\right)\right)} .
$$

It is straightforward to show that $\pi_{t}$ decreases in $\mu_{t}$; since $\mu_{t+1}<\mu_{t}, \pi_{t}>$ $\pi_{t+1}$. Moreover, it converges to 1 as $\mu_{t} \downarrow \mu^{*}$. Therefore, the candidate for an equilibrium strategy for the $B$-buyer involves $\sigma\left(A \mid\left(h^{t}, p_{h}\right), \theta, B\right)=\pi_{t}$ if $t>\hat{t}$.

To finish the characterization of the PBE, notice that if the $B$-buyer randomizes with $\pi_{t}$, then given the beliefs of the seller, the seller is indifferent about switching at any $t \geq \hat{t}$. Similarly, given the seller's behavior strategy, the payoff from accepting $p_{h}$ in any meeting is the same as the option value of waiting. Therefore, the strategies constitute a PBE once they are complemented with the following beliefs that are derived using Bayes' rule:

$$
\mu_{t}= \begin{cases}\frac{(1-\alpha)^{t} \mu_{0}}{\mu_{0}(1-\alpha)^{t}+\left(1-\mu_{0}\right)} & \text { if } t<\hat{t} \\ \frac{\left((1-\alpha)+\alpha\left(1-\pi_{t}\right)\right)^{t-\hat{t}} \mu^{* *}}{\mu^{* *}\left((1-\alpha)+\alpha\left(1-\pi_{t}\right)\right)^{-\hat{t}}+\left(1-\mu^{* *}\right)} & \text { if } t \geq \hat{t} .\end{cases}
$$

This completes the characterization of the PBE described in example 2.

Proof of Proposition 6. The PBE described in Proposition 5 exists for $\delta \geq \delta^{*}$. Fix $\beta$ and let $\delta \rightarrow 1$; given the seller's strategy, the strategies of the buyers continue to be optimal in the limit. Now let $\beta \rightarrow 1$; given the behavior of the buyers, the seller cannot improve her payoff by posting $p_{h}$, so always $p_{l}$ ' remains optimal despite the fact that the cost of waiting becomes negligible. The result is the same if we first fix a value of $\delta \geq \delta^{*}$ and reverse the order in which the limits are taken.

Proof of Proposition 7. (i) The PBE described in Proposition 3 (ii) exists for $\beta \geq \beta^{*}$. Fix $\beta$ and let $\delta \rightarrow 1$; given that the seller always posts $p_{h}$, the strategies of the buyers continue to be optimal in the limit. Now let $\beta \rightarrow 1$; given the behavior of the buyers, it is obvious that the seller's strategy remains optimal in the limit. The result is the same if we first fix a value of $\delta$ and reverse the order in which the limits are taken. For if $\beta \rightarrow 1$ then, given the behavior of the buyers, the seller will continue to find it optimal to post $p_{h}$ in every period. But then $B$-buyers cannot improve their payoffs by rejecting $p_{h}$; hence, their strategies remain optimal as $\delta \rightarrow 1$.

(ii) Recall that, for given values of $\beta$ and $\delta$, the seller announces $p_{h}$ if his belief is above $\mu^{*}$, and $p_{l}$ otherwise. Fix $\delta$ and let $\beta \rightarrow 1$; then $\mu^{*} \rightarrow 0$ by Proposition 4 (ii) and the fact that $\beta^{*}=1$ in this case; that is, in the limit, the seller always announces $p_{h}$ in period one. Now let $\delta \rightarrow 1$; since $\delta^{*} \geq 1$, it follows that accepting a high price is at least as good as rejecting it, so the 
strategy of $B$-buyers continues to be optimal in the limit. Therefore, if the state is $H$, then the seller trades in the first period at $p_{h}$; if the state is $L$, she observes a rejection in the first period and trades at $p_{l}$ in the second. That is, she always trades at the right price. An argument similar to the one used in (i) reveals that the result is the same if we reverse the order in which the limits are taken.

\section{References}

Bergemann, D., Valimaki, J.: Learning and strategic pricing. Econometrica 65, 1125-1149 (1996)

Blouin, M., Serrano, R.: A decentralized market with common values uncertainty; non-steady states. Department of Economics, Brown University, mimeo (1998)

Datta, M., Mirman, L., Schlee, E.: Optimal experimentation in signal dependent decision problems. Department of Economics, Arizona State University, mimeo (1998)

Easley, D., Kiefer, N.: Controlling a stochastic process with unknown parameters. Econometrica 56, 1045-1064 (1988)

Lazear, E.: Retail pricing and clearance sales. American Economic Review 76, 14-32 (1986)

Mirman, L., Samuelson, L., Urbano, A.: Monopoly experimentation. International Economic Review 34, 549-564 (1993)

Nyarko, Y. On the convexity of the value function in Bayesian control problems. Economic Theory 4, 303-309 (1994)

Rothschild, M.: A two-armed bandit model of market pricing. Journal of Economic Theory 9, 185202 (1974)

Royden, H.: Real analysis. New York: Macmillan 1988

Serrano, R., Yosha, O.: Information revelation in a market with pairwise meetings: the one-sided case. Economic Theory 3, 481-499 (1993)

Serrano, R., Yosha, O.: Welfare analysis of a market with pairwise meetings and asymmetric information. Economic Theory 8, 167-175 (1996)

Taylor, C.: Time-on-the-market as a sign of quality. Review of Economic Studies 66, 555-578 (1999)

Wolinsky, A.: Information revelation in a market with pairwise meetings. Econometrica 58, 1-23 (1990) 